\title{
MISE EN OEUVRE D'OUTILS OPEN SOURCE POUR LE SUIVI OPÉRATIONNEL DE L'OCCUPATION DES SOLS ET DE LA DÉFORESTATION À PARTIR DES DONNÉES SENTINEL RADAR ET OPTIQUE : ÉTUDES DE CAS EN GUYANE ET AU TOGO
}

\author{
Cédric Lardeux ${ }^{1}$, Anoumou Kemavo ${ }^{1}$, Maxence Rageade ${ }^{1}$, Mathieu Rahm¹, \\ Pierre-Louis Frison ${ }^{2}$, Jean-Paul Rudant ${ }^{2}$ \\ 1: ONF International, 45 Avenue de la Belle Gabrielle, 75012 Paris, France \\ 2: Universite Paris-Est, LaSTIG UPEM / IGN, 5 bd Descartes, 77454 Marne-la-Vallee, France
}

\begin{abstract}
Résumé
La télédétection est un outil particulièrement adapté pour le suivi de l'occupation des sols en général ; il est aussi particulièrement utilisé pour le suivi de la déforestation. De par l'envoi des satellites Sentinel-1 et Sentinel-2 via le programme Copernicus, la communauté dispose maintenant de données gratuites, avec des périodes de revisites temporelles réduites, permettant au plus grand nombre de suivre efficacement la dynamique d'occupation d'une zone d'étude. Ce papier pré-sente une méthode de suivi de l'occupation du sol se basant sur la combinaison de deux approches s'appuyant sur des outils logiciels Open Source (QGIS, Orfeo ToolBox, python). Nous combinons tout d'abord le suivi de l'occupation du sol à une échelle de temps annuelle utilisant des données Sentinel-1 et Sentinel-2 puis une approche de suivi des change-ments liés à la déforestation à une échelle de temps bi-mensuelle à partir des données Sentinel-1. Les résultats obtenus démontrent la bonne synergie de ces approches, qui permettent d'utiliser de façon complémentaire les données optiques et radar. En vue de rendre accessible la méthode proposée, tous les outils Open source utilisés sont disponibles sur ce lien : http://remotesensing4all.net/index.php/2018/09/11/kitdutilisation-des-donnees-radar-sentinel-1-lors-de-latelier-radar-du-foss4g-fr-2018-2/.
\end{abstract}

Mots clés : Télédétection, Sentinel-1, Sentinel-2, Optique, Radar, Classification, Déforestation, Séries temporelles, Togo, Guyane française

\begin{abstract}
Remote sensing is a particularly suitable tool for monitoring land use but also particularly suited for deforestation monito-ring. By launching the Setinel-1 and Sentinel-2 satellites in the Copernicus program, the community now has free data with important time-revisits allowing the greatest number of people to effectively monitor land cover of a study area. This paper presents a land use monitoring method based on the combination of two approaches computed using Open Source tools (QGIS, Orfeo ToolBox, python). First, we focus on land use monitoring at an annual time scale using Sentinel-1 and Sentinel-2 data, and then, by the use $f$ entienl-1 data, we detect changes in the forest cover due to deforestation at bi-monthly time scale. The results obtained show a very good synergy of these approaches allowing the complementary of optical and radar data. In order to make accessible the proposed method, all the used open source tools are available on this link http ://remotesensing4all.net/index.php/2018/09/11/ kit-use-des-donnees-radar- sentinel-1-in-de-latelier-radar-du-FOSS4G-en-2018-2 /.
\end{abstract}

Keywords : Remote sensing, Sentinel-1, Sentinel-2, Optic, Radar, Classification, Deforestation, Time series, Togo, French guiana

\section{Introduction}

La télédétection est un outil privilégié pour le suivi des paysages naturels, notamment grâce aux observations continues sur de grands espaces, répétitives sur de longues périodes. Depuis leur apparition dans les années 70 , les données de télédétection optique ont été largement utilisées pour l'observation de la Terre. En raison de la couverture nuageuse quasi permanente sur les régions tropicales, l'observation dans le spectre optique (visible-proche et moyen infra rouge) est difficile, ce qui représente l'une des limitations principales des données acquises dans ce domaine spectral. Par ailleurs, depuis 1991 et le lancement du satellite ERS-1, des données radar sont acquises en continu à l'échelle globale. Le panorama s'est considérablement enrichi depuis, avec la mise en orbite d'autres Radar à Synthèse d'Ouverture (RSO) permettant de générer des images des surfaces observées avec des résolutions spatiales allant du mètre à la trentaine de mètres. En raison des longueurs d'ondes centimétriques utilisées $(2 \mathrm{~cm}, 6 \mathrm{~cm}, 24 \mathrm{~cm}$ respectivement pour les bandes $\mathrm{X}, \mathrm{C}, \mathrm{L})$, l'intensité du signal radar est peu perturbée par l'atmosphère, ce qui permet l'observation des surfaces terrestres même en présence de 
couverture nuageuse.

Depuis 2014, le programme Copernicus de l'Union Européenne a permis la mise en orbite de nouveaux satellites radar et optique, de résolutions spatiales décamétriques, respectivement Sentinel-1A et $1 \mathrm{~B}$ et Sentinel$2 \mathrm{~A}$ et $2 \mathrm{~B}$. Ces satellites ouvrent une nouvelle ère dans le suivi par télédétection de la surface terrestre de par l'accès à des données gratuites acquises tous les 6 jours et 5 jours, respectivement pour Sentinel- 1 et Sentinel2 , capteurs sur lesquels nous allons porter notre attention dans cet article. La cartographie à partir d'images satellites est ainsi un outil particulièrement adapté pour obtenir, d'une part une vue d'ensemble de l'occupation du sol d'une zone géographique à une date donnée, et d'autre part pour en réaliser le suivi temporel. Ce type d'analyse s'avère utile pour un grand nombre d'applications. Citons par exemple, le suivi de la déforestation liée à l'exploitation forestière, le suivi de la croissance et la certification de plantations, l'implantation de projets d'agro-foresterie. Parallèlement, ces techniques de cartographie par imagerie spatiale sont particulièrement utilisées dans le cadre de projet REDD+ (Réduction des émissions issues de la déforestation et de la dégradation forestière) comme le montre l'une des méthodologies couramment utilisées, VM0015 («Methodology for Avoided Unplanned Deforestation » v1.1 Partie 2, Chapitre 2) nécessitant de cartographier l'évolution du couvert forestier dans le passé.

Toutefois, malgré la grande richesse potentielle des données disponibles, leur usage s'avère parfois comple$x e$, soit parce que certains outils sont en première approche trop compliqués, soit, bien souvent, par manque d'expérience de l'utilisateur; manque d'expérience qui révèle une méconnaissance des outils et techniques accessibles. Deux exemples d'étude touchant au suivi de la dynamique forestière en zone tropicale humide sont présentés ici. Le premier, exploitant uniquement les données radar Sentinel-1, est dédié au suivi de la déforestation avec un pas de temps bi-mensuel. Le deuxième, utilisant conjointement les données optique et radar acquises par les satellites Sentinel-2 et Sentinel-1 est dédié au suivi temporel de l'occupation du sol, avec un pas de temps annuel. Cette étude s'avère donc moins exigeante que celle portant sur le suivi de la déforestation en termes de réactivité, mais à l'inverse, elle est plus exigeante en termes de précision. Ces études concernent respectivement la Guyane française et le Togo. En termes de données et logiciels, ces approches utilisent des images libres d'accès (Sentinel-1 et 2 ) et des outils de même nature «Open Source », notamment QGIS et OTB (Orfeo ToolBox). Certains de ces outils ont été aménagés afin de les rendre o pérationnels et exploitables $p$ ar des utilisateurs non spécialistes en informatique.

\section{Site d'étude}

\subsection{Guyane française}

En Guyane, la forêt, qui couvre plus de 8 millions d'hectares soit environ $96 \%$ du territoire, constitue un pa- trimoine exceptionnel qu'il s'agit de valoriser et de conserver. L'ONF (Office National des Forêts) a une responsabilité forte au regard de cet objectif en Guyane puisque l'établissement y assure, pour le compte de l'État, la gestion de près de 6 millions d'hectares de forêts domaniales.

Outre l'aménagement et la valorisation économique de ces forêts, l'ONF a pour mission la protection des milieux et des espèces, la surveillance du territoire et la gestion du foncier. Face à ces enjeux et à l'immensité du territoire à couvrir, l'ONF se repose principalement sur l'utilisation des outils de télédétection et sur une équipe d'agents assermentés qui ont pour mission de faire respecter la réglementation environnementale et foncière applicable en Guyane.

Dans le cadre de ses missions de surveillance et de protection des milieux, l'ONF a développé il y a dix ans une méthodologie de suivi de l'impact de l'activité minière sur le couvert forestier et les cours d'eau à partir de données satellites optiques. Les résultats de traitement de ces données permettent d'orienter efficacement les actions de l'Observatoire de l'Activité Minière (OAM) créé en 2008 à l'initiative de l'ONF, pour assurer le partage d'information entre toutes les institutions locales concernées par le suivi des activités d'orpaillage illégales. Toutefois, bien que l'utilisation des outils de télédétection ont fait leurs preuves dans le renforcement du contrôle de l'activité minière illégale mais aussi légale, la méthodologie basée sur l'utilisation de données optiques entraîne des limites opérationnelles : photo-interprétation visuelle chronophage, difficultés d'automatisation des chaînes de traitement dues à la sensibilité des capteurs optiques aux conditions atmosphériques, capacité d'observation du territoire et d'utilisation des données limitées par la présence de couverture nuageuse quasi permanente en Guyane.

Or, depuis dix ans, les pressions sur la forêt augmentent en Guyane, engendrant un besoin pour un suivi du territoire de plus en plus précis, exhaustif et fréquent, alors que l'ONF reste limité en capacités humaines. Ce besoin s'illustre notamment par la demande récente de la préfecture de Guyane pour la mise en place d'un Observatoire du foncier, à l'instar de l'OAM, suite à de nouveaux événements de déforestations illicites en 2016, sur plus de 90 hectares de terrain de l'état.

A l'instar des besoins, la technologie évolue. Depuis 2014, dans le cadre du programme européen Copernicus, les stellites Sentinel de l'Agence Spatiale Européenne fournissent en accès libre des données radar (Sentinel-1) acquises tous les six jours en Guyane. Ces données, capables de "voir à travers les nuages » et d'une grande stabilité, offrent des perspectives innovantes très intéressantes pour la mise en place d'un système de suivi quasi temps réel, semi-automatisé et exhaustif de la déforestation sur le territoire.

On se propose ainsi de présenter une approche opérationnelle du suivi de la déforestation à un pas de temps bi-mensuel afin d'en percevoir les qualités mais aussi les limites à l'échelle de toute la Guyane comme illustré par la figure 1. 


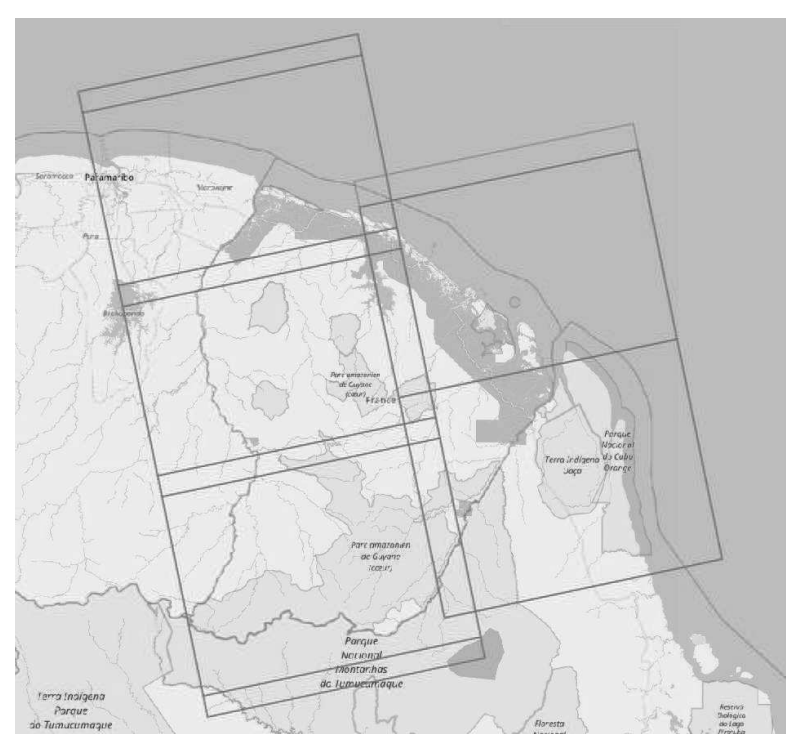

Figure 1 : Emprise des données Sentinel-1 utilisées pour le suivi de la déforestation. Durant l'année 2016, des acquisitions en orbite ascendante ont été réalisées à 57 dates différentes (26 dates pour l'orbite relative 47 et 31 dates pour l'orbite 120).

L'ensemble des données utilisées ont été acquises en 2016 dans le mode IW pour les polarisations VV et VH avec un niveau de produit GRD (Ground Range Detected). Pour faciliter l'analyse, nous avons travaillé uniquement avec des données en mode ascendant comportant le plus de dates disponibles, à savoir les orbites relatives 47 et 120 . Ces deux orbites permettent de couvrir toute la largeur de la Guyane française du 31/01/2016 au 20/12/2016 pour l'orbite 47 (26 dates) et du 05/02/2016 au 25/12/2016 pour l'orbite 120 (31 dates).

Afin d'analyser les résultats de la méthode de suivi de la déforestation, nous avons comparé visuellement les résultats aux données optiques lorsque celles-ci étaient disponibles (la couverture nuageuse souvent très importante). Par ailleurs un survol en hélicoptère accompagné d'une mission terrain au sol a appuyé l'analyse des résultats. Cette dernière s'est faite sur l'ensemble du territoire en se focalisant sur les principaux moteurs de la déforestation, à savoir l'agriculture et l'orpaillage. Les sites connus pour leur forte dynamique de changement ont été analysés et comparés plus finement.

\subsection{Togo}

Le Togo fait partie de l'écosystème forestier tropical sec. Cet écosystème est caractérisé par des reliques forestières, des forêts galeries et de vastes mosaïques de savanes boisées, de savanes arbustives, de savanes herbeuses et de zones sans végétation (sol nu, habitation et agglomération). On y trouve en particulier des espèces de décidus perdant leurs feuilles une partie de l'année. Cet écosystème est également caractérisé par une grande variabilité saisonnière (alternance de saisons des pluies et de saisons sèches), ce qui provoque de fortes variations de l'état du couvert végétal. En saison des pluies, les couverts végétaux des savanes boisées, arbustives, herbeuses présentent une forte activité chlorophyllienne, ce qui leur donne, sur les images, l'aspect de parcelles forestières. II s'ensuit une confusion entre ces différents couverts. La situation s'inverse pendant la saison sèche, où les zones de forêts et de savanes composées d'espèces décidues perdent leurs feuilles et prennent, sur les images, l'apparence de zone sans végétation. Ces variabilités saisonnières rendent alors complexe la cartographie par imagerie satellitaire. Cette partie consacrée au Togo, se propose d'explorer les potentialités des images du programme Copernicus Sentinel-1 et 2 (acquisition tous les 6 jours pour Sentinel-1 et tous les 5 jours pour Sentinel-2 avec un pixel de 10m) pour mettre en place une cartographie annuelle d'occupation du sol. Notre choix s'est porté sur la Réserve de Faune de Togodo (RFT) située entre $1^{\circ} 20$ et $1^{\circ} 40$ de longitude Est et entre $6^{\circ} 40$ et $6^{\circ} 50$ de latitude Nord entre les régions maritime et des plateaux au Sud à environ $150 \mathrm{~km}$ de la capitale Lomé, comme l'illustre la figure 2.

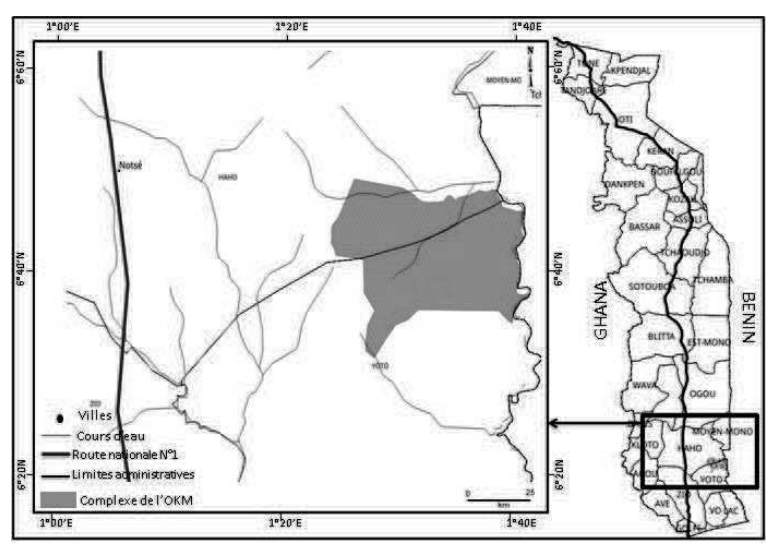

Figure 2 : Localisation de la réserve de faune de Togodo.

La RFT couvre une superficie t otale de 25500 ha et est composée d'îlots de forêts semi-décidues, de galeries forestières longeant les principaux cours d'eau, de vastes savanes (arbustives et herbeuses) de types souda-no-guinéennes. Cet ensemble homogène de savanes et forêts constitue un habitat idéal pour la faune diversifiée de la réserve. Ainsi le classement de cette zone en ré-serve de faune (statut particulier dans la législation fo-restière togolaise permettant sa protection) a pour but de protéger la diversité floristique e $t \mathrm{f}$ aunique. $\mathrm{L}$ a RFT, en tant que zone boisée proche des grandes villes du Sud (Lomé, Tsévié, Tabligbo, Kové), offre divers services socioculturels à la population : des activités récréatives, scientifiques e $t \mathrm{t}$ ouristiques, I e $r$ amassage $\mathrm{d}$ e $\mathrm{b}$ ois de chauffe et la fourniture de forestiers non ligneux. Cependant plusieurs menaces pèsent sur la RFT (l'agriculture itinérante, le pâturage du bétail, la coupe d'espèces précieuses et rares et la surexploitation des produits forestiers non ligneux), et mettent en péril sa survie et la pérennité des services qu'elle offre. La protection, la sauvegarde et la conservation de cet écosystème fragile s'avèrent donc indispensable. 
Un des moyens pour assurer la sauvegarde et la conservation des ressources fragiles de la RFT est de mieux connaître l'état des lieux de ses ressources et d'être capable d'en assurer un suivi régulier et annuel. La cartographie par imagerie satellitaire comme il a été dit précédemment présente un avantage certain et majeur. Pour réaliser la cartographie annuelle de l'occupation du sol de la RFT et mettre en place une approche de suivi annuel des modes d'occupation du sol, nous avons utilisé une série temporelle de 33 images Sentinel-1 acquises entre le 21-12-2015 et 03-01-2017 dans le mode IW (polarisations VV et VH) et 5 images Sentinel-2 acquises sur la même période (sélection d' images optiques de couverture nuageuse inférieure à 10\%) comme l'illustre la figure 3.

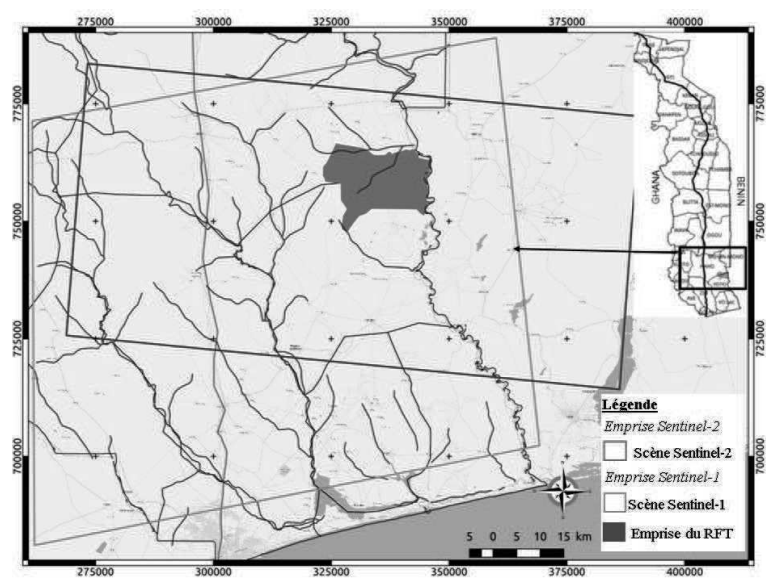

Figure 3 : Emprise des images Sentinel-1 et 2 sur le site de RFT au Sud-Togo.

\section{Méthodologie}

L'utilisation de l'imagerie spatiale pour réaliser le suivi de l'occupation du sol nécessite quelques prétraitements génériques que nous mentionnerons rapidement dans ce paragraphe. Nous poursuivrons ensuite l'exposé en présentant l'outil de classification utilisé pour réaliser des cartes d'occupation du sol.

\subsection{Pré-traitements des données}

\subsubsection{Données optiques}

II s'agit ici essentiellement de la technique dite de «pan-sharpening» a les bandes spectrales sont fusionnées afin que l'ensemble des images soit disponibles avec la taille de pxel correspondant aux canaux les reux résolus. Dans le cas de Sentinel-2, il s'agit des canaux 2 $3,4,8$ (plus de détails sur les longueur d'ondes et resolution des différentes bandes sur ce lien https : //earth - .esa.int/web/sentinel/user-guides/sentinel-2-msi - /resolutions/spatial) avec un pxel de $10 \mathrm{mx} 10 \mathrm{mCette}$ procédure facilite l'analyse visuelle par composi-tion trichrome de tous les triplets possibles à partir des 13 bandes spectrales initiales. Pour ce pétraitement, rous a-vons utilisé l'algorithme de Brovey implémenté sous l'OTB, Maglione et al. (2016).

\subsubsection{Données radar}

Les données Sentinel-1 utilisées sont au format GRD, mode IW, double polarisation VV et HV (plus de détails sur les format et résolution des données Sentinel-1 sur ce lien https : //sentinel.esa.int/web/sentinel/user guides/sentinel-1-sar/resolutions/level-1-groundrange - detected). La chaîne de traitement a été développée à partir de l'OTB et est disponible sous QGIS via un script python, explicité par la figure 4.

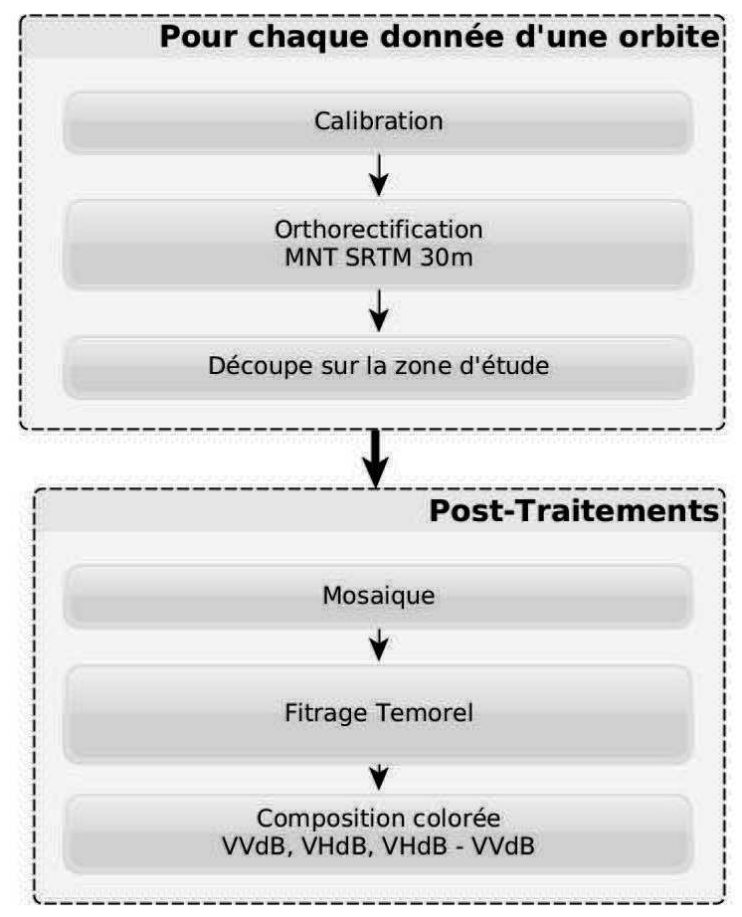

Figure 4 : Prétraitements des données Sentinel-1.

Cette chaîne de traitement concerne chaque image de la série temporelle utilisée. Sont successivement appliquées : une calibration en gamma 0 (terrain relativement plat), une orthorectification (utilisant I e m odèle numérique de terrain SRTM au pas de $30 \mathrm{~m}$ ), puis un découpage uniquement sur la zone d'étude afin d'obtenir une parfaite superposition des données. Pour chaque date d'acquisition, l'ensemble des données donne ensuite lieu à la création d'une mosaïque. Afin de réduire le chatoiement radar, nous utilisons un filtre temporel Quegan et Yu (2001). Pour faciliter l' analyse des résultats, chaque date donne lieu à une composition colorée $\mathrm{R}$ (VV $\mathrm{dB}), \mathrm{V}(\mathrm{VHdB}), \mathrm{B}(\mathrm{VHdB}-\mathrm{VVdB}$, comme l'illustre la figure 5 .

\subsection{Suivi de la déforestation sur la Guyane}

La méthode proposée est basée sur la haute fréquence de revisite temporelle des données Sentinel-1 (délai de revisite de 6 jours) associée à leur capacité à «voir à travers les nuages» pour développer un système d'alerte quasi temps réel, robuste, exhaustif et économique en terme de coût (images gratuites) et de temps de travail (automatisation de la chaîne de traitement). Concernant l'automatisation, des outils ont été conçus 


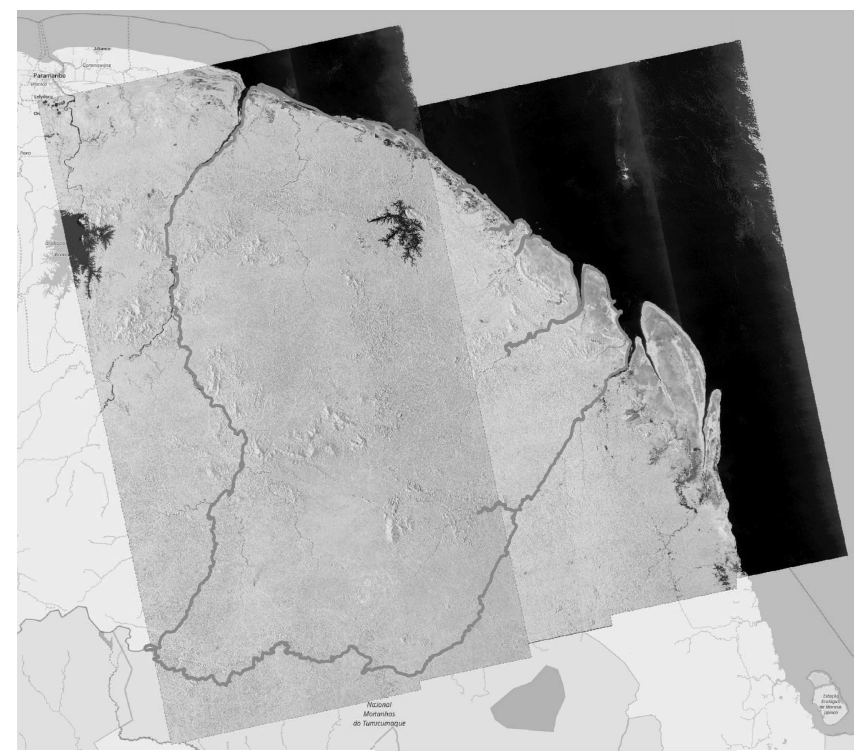

Figure 5 Composition colorée, Rouge VV dB - Vert VH $d B$ - Bleu VH-VV $d B$, obtenu après traitement sur deux données acquises en décembre 2017.

spécifiquement pour leur utilisation dans le logiciel libre QGIS afin de facilité leur diffusion.

La méthode développée comporte trois grandes étapes:

1. Prétraitement des données (calibration, orthorectification, filtrage temporel) évoqué dans le chapitre 3.1.2.

2. Optimisation du contraste Forêt / Zone déforestée, notamment par l'atténuation de l'effet saisonnier sur la radiométrie

3. Déclenchement d'une alerte suite à l'analyse de la variation de la radiométrie dans le temps, en réponse à un phénomène de changement ou de déforestation provoquant une forte variation de radiométrie $(>1 \mathrm{~dB})$ au cours du temps.

Cette méthode analyse dans son ensemble la série temporelle issue des données Sentinel-1. Nous choisissons d'utiliser uniquement la polarisation $\mathrm{VH}$ car cette polarisation est plus sensible à la densité de végétation que la polarisation VV, Mermoz et Le Toan (2016). Ainsi, une baisse significative de la réponse $\mathrm{VH}$ pourra correspondre à une surface déforestée.

La figure 6(1) présente les variations de la réponse $\mathrm{VH}$ (en $\mathrm{dB}$ ) en fonction du temps sur différents sites d'étude connus pour avoir été déforestés à des instants différents. L'analyse des résultats obtenus sur ces 4 sites montre bien à un instant donné une baisse marquée de la radiométrie ; par exemple aux dates $t_{5}$ et $t_{11}$, respectivement pour les sites 1 et 2 à 4 . Une baisse existe également sur le site 3 , mais très modérée. Les baisses les plus nettes correspondent au phénomène de déforestation. En revanche, on remarque qu'avant ou après ces différents évènements, la réponse du signal n'est pas constante. La variation de la radiométrie dans le temps pour une zone donnée semble correspondre à des effets saisonniers, lesquels risquent de venir perturber nos analyses. Ainsi, l'humidité de surface et la densité de végétation peuvent varier au cours du temps et générer des variations de rétrodiffusion au sein de surfaces que l'on juge par ailleurs stables en termes d'occupation du sol.

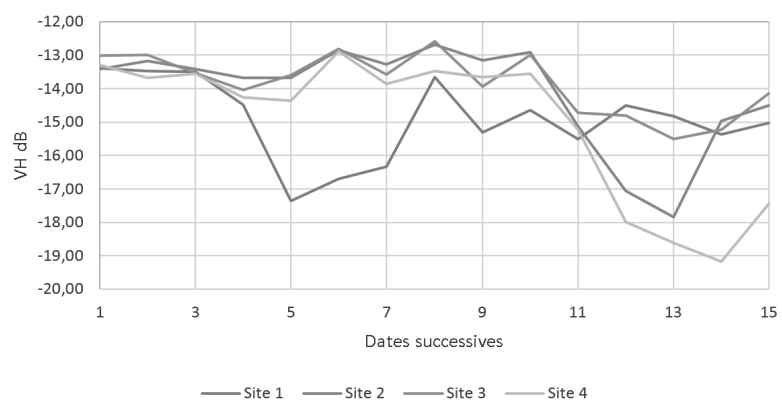

(1)

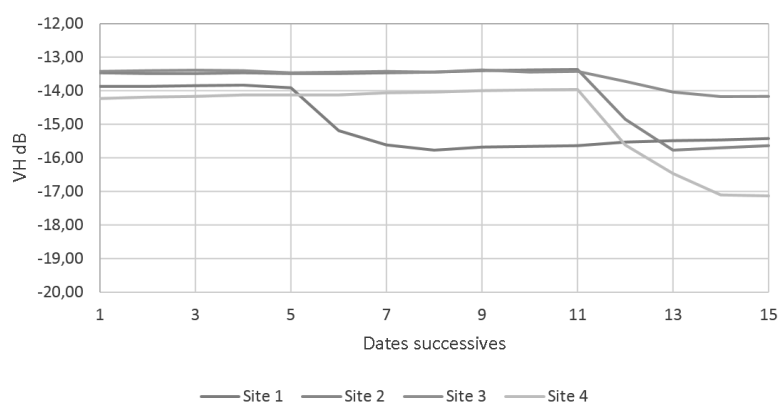

(2)

Figure 6 : Variation de la radiométrie de la polarisation $\mathrm{VH}$ (en décibels) en fonction du temps (15 dates entre janvier et dé-cembre 2016). Chaque courbe est relative à un site ayant subi différents types de déforestation (par petit patch pour le site 3 et une coupe rase pour les autres sites. Déforestation en date 5 pour la courbe bleue, date 12 pour la courbe orange, grise et jaune). La figure 6(1) représente la série temporelle en l'ab-sence de post traitement alors que la figure 6(2) présente le résultat obtenu après application de la méthode d'optimisation du contraste (traitement 1 et 2) entre les surfaces de forêt et de non forêt.

Comme le montre la figure 6(1), ces effets perturbateurs sont moins marqués sur les surfaces forestières que sur les surfaces ayant une densité de végétation moindre, respectivement avant et après la baisse de la radiométrie. En effet, plus la végétation est dense et plus on se rapproche du phénomène de saturation du signal. Le site 3 représenté par la courbe grise de la figure 6(1) est un site ayant subi une déforestation par petit patch alors que les autres sites ont subi une déforestation se rapprochant d'une coupe rase.

Afin de réduire les effets évoqués précédemment et ainsi limiter les fausses alarmes, nous appliquons successivement les deux traitements suivants :

- Traitement 1 - Ajustement des niveaux radiométriques de la série temporelle à partir de la radiométrie de référence estimée sur des zones de forêt dense et stable sur la série temporelle.

- Traitement 2 - Filtre passe-bas du premier ordre 
des profils temporels.

Le premier traitement consiste à considérer que la réponse d'une forêt dense humide sans changement anthropique est stable dans le temps. Afin d'ajuster les niveaux radiométriques de la série temporelle sur l'ensemble de la scène d'étude, nous estimons pour chaque date $t$ une radiométrie de référence, $\widehat{\gamma_{0}}(t)$. Cette intensité de référence est ici calculée à partir de polygones correspondants à des surfaces de forêt dense stables sur l'ensemble de la série temporelle. Afin de permettre un suivi régulier, il est conseillé d'utiliser un grand nombre de polygones (un minimum de 10 polygones comportant chacun au moins 1000 pixels) sur l'ensemble de la zone afin de ne pas biaiser la moyenne si l'une de ces surfaces venait à être déforestée. On calcule alors le niveau de référence moyen sur l'ensemble de la série temporelle, $<\widehat{\gamma_{0}}(t)$ Ref $>$. Pour chaque pixel de l'image on calcule alors

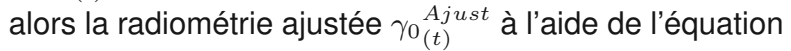
suivante :

$$
\gamma_{0}^{\text {Ajust }}=\frac{\gamma_{0}(t)}{\widehat{\gamma}_{0}(t)} \cdot<{\widehat{\gamma_{0}}}_{(t)}^{\text {Ref }}>
$$

où $\gamma_{0(t)}$ représente la radiométrie d'un pixel à la date $t$.

La deuxième étape du traitement est appliquée après réajustement radiométrique destiné à éliminer les variations saisonnières. Cette étape consiste à appliquer un filtre passe-bas du premier ordre à $\gamma_{0}{ }_{(t)}^{\text {Ajust }}$ sous la forme d'une moyenne pondérée exponentielle à la série temporelle NIST (2018). Cette moyenne pondérée exponentielle appliquée à chaque pixel à la date $t, \widehat{\gamma}_{(t)}^{\text {Ajust }}$ se calcule de la façon itérative suivante $(t=0$ correspond à la première date de la série temporelle) :

$$
{\widehat{\gamma_{0}}}_{(t)}^{\text {Ajust }}=\left\{\begin{array}{cc}
\gamma_{0}^{\text {Ajust }} & , \quad t=0 \\
\alpha \cdot \gamma_{0}{ }_{(t)}^{\text {Ajust }}+(1-\alpha) \cdot \widehat{\gamma}_{(t-1)}^{\text {Ajust }} & , \quad t>1
\end{array}\right.
$$

où $\alpha$ représente un coefficient pondérateur entre deux dates, compris entre 0 et 1 .

Cette technique consiste ainsi à réaliser pour un pixel donné une moyen-ne (pondérée) temporelle cumulée dans le temps.

L'intérêt pour réduire la remontée de la radiométrie après déforestation liée à l'effet saisonner (cf courbe bleue de la figure 6(1)) consiste à pondérer la moyenne en donnant un poids moins important à la nouvelle date, en utilisant par exemple une valeur de $\alpha=0,3$ (valeur empirique retenue pour l'intérêt des résulats obtenus).

La contre partie de cette technique est d'introduire un retard de détection de la déforestation en raison de l'intégration des dates antérieures

La figure 6 montre qu'une fois la méthode d'optimisation du contraste appliquée, la réponse temporelle observée avant et après le phénomène de déforestation se trouve fortement lissée. Par ailleurs, le phénomène saisonnier qui occasionne une augmentation progressive de la radiométrie, est très fortement atténué (figure 6(2)).
Une fois l'optimisation du contraste réalisée nous appliquons une méthode d'analyse des variations de la radiométrie pour déclencher une alerte de déforestation. Ainsi, une surface sera identifiée comme déforestée à la date $t$ si elle respecte les deux règles suivantes :

1. $s_{t}^{0}-s_{t_{r} e f}^{0}<$ seuil 1

$s_{t_{r} e f}$ étant la première date de la série tempo-relle.

La valeur de seuil1 a été fixée à $-1,3 \mathrm{~dB}$ (ligne pointillée rouge sur la figure $7(1)$ ) après analyse de différents cas de déforestation rapide et rase.

2. Pour une date t retenue à l'étape 1 , celle-ci sera validée si $s_{t} \underline{0} s_{t-1}^{0}<$ seuil2. La valeur de Seuil 2 a été fixée à $-0.5 \mathrm{~dB}$ (ligne pointillée rouge figure $7(2)$ ). Ce seuil car bien adapté à un changement rapide et important.

En effet, l'un des objectifs de la méthode est d'obtenir le moins possible de fausses alarmes. Le premier seuil permet ainsi de caractériser le changement sur une longue période alors que le deuxième seuil a pour rôle de réduire les fausses alarmes résiduelles en s'assurant de conserver les changements les plus importants sur une échelle de temps plus courte (entre deux dates successives).

Ces paramètres peuvent et doivent être ajustés en fonction de la zone d'étude et de la dynamique de déforestation. En effet, dans le cas de déforestation de type coupe rase, on utilisera des valeurs de seuil plus importantes que lors de déforestation progressive se traduisant par une baisse de la radiométrie plus progressive pouvant par ailleurs être moins importante. Cette méthodologie permet alors de produire une carte de déforestation incluant la date de détection supposée accompagnée d'une couche indiquant l'amplitude maximale de la chute de radiométrie.

\subsection{Outil de classification sur la réserve de Faune de Togodo}

Pour la cartographie des différents modes d'occupation du sol sur notre site d'étude au Togo, nous avons choisi d'utiliser l'algorithme de classification supervisée SVM (Support à Vastes Marge). Cet algorithme consiste à trouver l'hyperplan de séparation optimal entre les classes d'étude. Cette approche, qui s'affranchit de l'établissement à priori d'hypothèses sur la distribution statistique des données, permet d'utiliser et combiner des données aussi diverses que les données optiques et radar. Par ailleurs, l'utilisation des noyaux permet de séparer des classes dites non linéairement séparable. Plus de détails sur l'algorithme SVM sont donnés dans Lardeux et al. (2009) et Burges (1998).

Pour estimer le potentiel de contribution des images optiques et radar, les classifications sont réalisées séparément sur les images optiques et sur les images radar, puis en combinant ces 2 types de données. Toutes les classifications sont ensuite accompagnées d'indicateurs de validation de classification (précision utilisateur (PU), précision producteur (PP) et précision globale (PG)) Olofsson et al. (2014). 


\section{Résultats}

\subsection{Suivi de la déforestation}

Les résultats obtenus sont présentés figure 9.
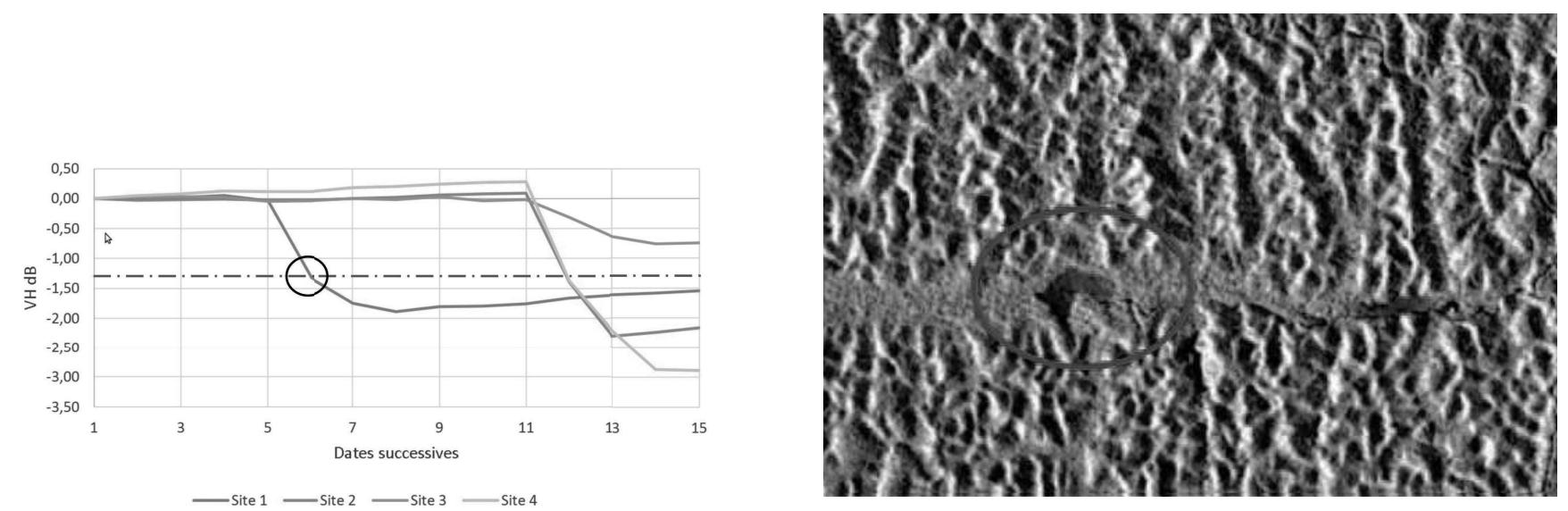

(1)

(1)

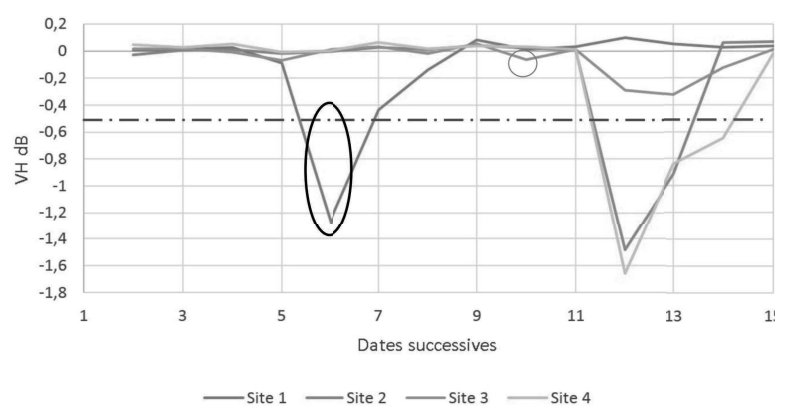

(2)

Figure 7 : Courbes illustrant les deux indicateurs de change-ments utilisés pour détecter le phénomène de déforestation (i.e. la différence entre la radiométrie à la date $t$ et celle à une date de référence $t_{\text {ref }}$ ). Chaque courbe colorée correspond à un site de déforestation spécifique. La figure $7(1)$ représente pour chaque date la différence en $d B$ entre la radiométrie au temps $t$ et celle correspondant au départ de la série temporelle. La figure 7(2) représente pour chaque date la différence entre mesures à la date $t$ et à la date ( $t-1)$. Les lignes pointillées visualisent les seuils utilisés pour chacun des indicateurs de changement. Le cercle noir pointe sur la date 6 où l'application de ces seuils permet de détecter la déforestation du site 1. 
Ainsi, la composition colorée multi-date, 8(1), basée sur la polarisation $\mathrm{VH}$ en décibels (rouge : août, vert : juillet et bleu : décembre) illustre parfaitement le poten-tiel de détection de la déforestation. Celle-ci fait appa-raître en magenta ou en bleu les différences importante de radiométrie observée sur les trois dates. La figure 8(3) représentant la polarisation VH (en décibels) au cours du temps, permet d'anlyser plus finement les évolutions temporelles de radiométrie observées. Les lignes colorées superposées à la courbe de radiométrie représentent les dates utilisées dans la composition colorée de la figure $8(1)$. Cette figure montre bien que le phénomène de déforestation est visible à partir du mois de septembre par une baisse significative de la radiométrie d'environ 1 décibel. En appliquant les réglages illustrés précédemment par la figure 6, l'approche permet de bien détecter la déforestation comme l'illustre la figure 8(2) où le code couleur utilisé permet de suivrela progressivité de l'évenement.

Bien que l'approche semble efficace lorsque la déforestation est brutale (passage de forêt à sol nu sans résidu au sol) et que les pentes sont faibles; une topographie marquée, des restes de bois ou de feuillage au sol, ou une autre modification de l'état de la surface font que l'approche atteint ses limites. Par exemple, les divers degrés d'avancement du chantier d'orpaillage légal (figure 9) conduisent à percevoir la déforestation en plusieurs étapes successives.

La zone en cours d'orpaillage est tout d'abord défrichée (photo de la figure $9(1)$ ), repérée par l'ellipse rouge sur les figures 9(3) et 9(2), présentant respectivement les images Sentinel-2 et Sentinel-1 de la zone. A ce stade, le sol peut être recouvert de troncs ou branches ayant une radiométrie radar plus importante (teinte jaune de la donnée Sentinel-1 dans l'ellipse rouge) que pour un sol complètement nu (teinte noire/marron de la donnée Sentinel-1 (ellipse bleue). Le contraste est ainsi peu marqué en comparaison de la forêt environnante (teinte jaune et cyan clair). Par la suite, une fois le sol totalement défriché, la zone est inondée comme l'illustre la figure 9(1) (photo de droite) avec l'ellipse bleue. Par conséquent et comme le prouve la figure 9(4) superposant le résultat de détection de la déforestation à une données Sentinel-2, la pointe de la zone déforestée (elipse rouge) n'est pas détectée en raison de la radiométrie encore élevée à ce stade de l'orpaillage.

\section{Conclusion}

L'analyse des résultats s'est révélée concluante avec une très bonne capacité de détection pour des surfaces généralement supérieures ou égales à 1 ha. Ce seuil de 1 ha est fixé de manière à sur détecter le moins possible. En effet, bien qu'il soit possible de détecter des zones de déforestation de superficie inférieure à 1 ha avec la méthode actuelle, le niveau de confiance et de précision du système commence à fortement chuter sous ce seuil. $A$ l'avenir, avec la mise opérationnelle de ce système, l'expérience nous permettra de fournir une estimation fine de ce niveau de précision. Cette limite actuelle, liée à la résolution des images Sentinel-1 $(20 \mathrm{~m}$ de résolution spatiale pour un pixel de $10 \mathrm{~m}$ ), impacte la qualité de dé-

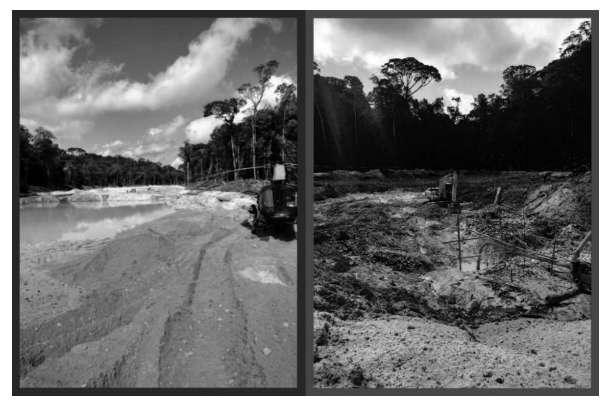

(1)

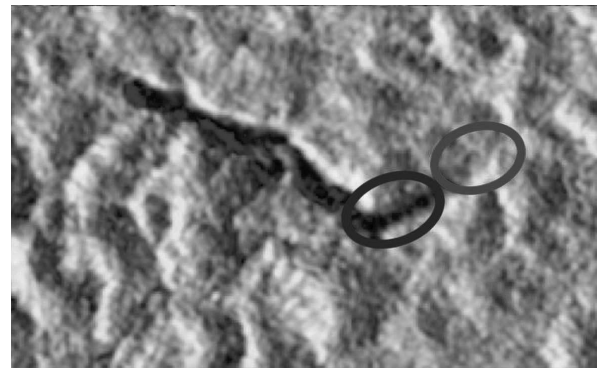

(2)

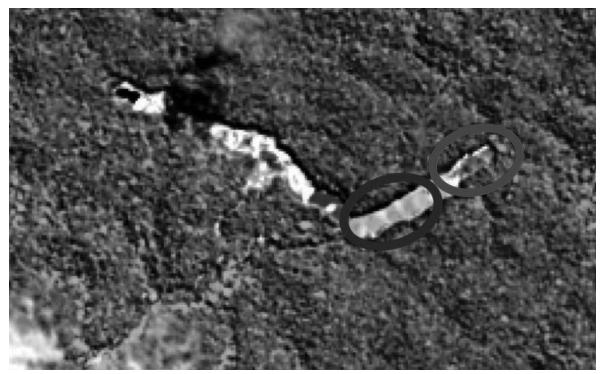

(3)

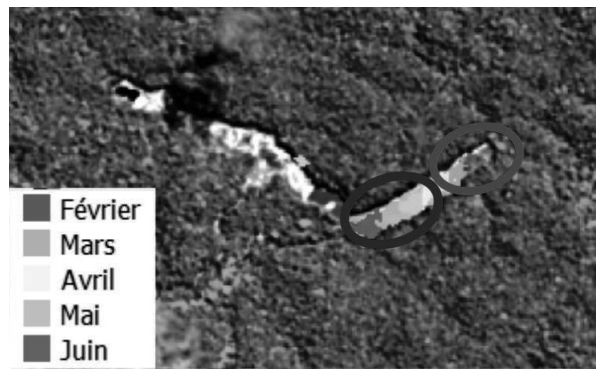

(4)

Figure 9 : Les photos de terrain en (1) encadrées en bleu et rouge représentent respectivement une zone de décantation et une zone avant inondation. Ces deux zones sont localisées par des cercles bleu et rouge sur les illustrations (2) et (3) super-posés respectivement à une donnée Sentinel-1 acquise le 17-06-2017 (canaux $R G B$ respectivement $\mathrm{VV}, \mathrm{VH}$ et $\mathrm{VH}-\mathrm{VV}$ ) et une données Sentinel-2 acquise le 16-06-2017 (canaux RGB respectivement Moyen Infra-Rouge, Proche Infra Rouge et Rouge). La figure 4 superpose le résultat de la détection de la défores-tation à partir des données Sentinel-1 sur la donnée Sentinel-2.

tection de l'orpaillage clandestin qui s'opère bien souvent sous couvert et sur de faibles surfaces (on parle alors généralement de dégradation forestière plutôt que de déforestation). De plus, la méthode peut également présenter certaines limites lorsque la zone déforestée est fortement 
accidentée ou soumise à une forte topographie, ce qui est surtout le cas pour les petites surfaces voisines de 1 ha. Par conséquent, la méthode présentée ci-dessus doit être considérée comme un système d'alerte quasi temps réel permettant de suivre finement les phénomènes de déforestation pour des surfaces de l'ordre ou supérieures a 1 ha. Par ailleurs, pour ce qui est du suivi des activités d'orpaillage illégal sous couvert ou pour la quantification précise des su rfaces dé forestées, il s 'avère nécessaire de compléter l'analyse avec de la donnée optique de type Sentinel-2. Le système d'alerte radar nous permettra d'une part de diminuer les besoins de recourir aux images optiques, et d'autre part de préciser la localisation des zones ou des analyses complémentaires sont nécessaires. A ce jour, au regard des résultats et en l'état des développements réalisés, la méthode peut être considérée comme opérationnelle pour le contexte guyanais, ce qui représente une avancée technologique importante à l'échelle globale dans le domaine du suivi de la déforestation en milieu tropical. En exploitant le potentiel de cette méthode, la forêt de Guyane pourrait devenir l'une des forêts tropicales les mieux surveillées au monde.

Bien qu'opérationnelle, la méthode doit toutefois encore être confrontée à un usage quotidien, afin notamment d'améliorer sa mise en oeuvre et son efficacité en situation réelle à travers les pistes d'amélioration déjà identifies. En effet, la technique étant basée sur la détection des changements qui induisent une chute de la radiométrie, de fausses alarmes peuvent apparaître. Comme ces fausses alarmes sont pour la plupart localisées en zone de non-forêt où la dynamique de changement est la plus forte, produire un masque de forêt est une étape nécessaire permettant de se focaliser uniquement en zone forestière et ainsi éliminer les fausses alarmes situées hors des zones d'intérêt. Autre amélioration prévue : l'automatisation de la chaîne de traitement avec des seuils d'alarme ajustés selon les retours d'expérience; utilisation des images en orbites Ascendantes et Descendantes afin d'optimiser l a d étection e $\mathrm{n} z$ one à relief marqué ; enfin réalisation des traitements avec des pixels de $10 \mathrm{~m}$ conduisant à plus de détails.

\subsection{Carte d'occupation du sol sur la réserve de Faune de Togodo}

Après interprétation visuelle des images Sentinel-1 (S1) et Sentinel-2 (S2), il a été identifié 8 classes d'occupation du sol illustrées par le tableau 4.2 présentant des vignettes pour les classes et capteurs considérés ( $\mathrm{S} 1$ et S2). Nous avons retenu les classes suivantes : forêts denses, plantations, savanes boisées, mosaïques de savanes arborées / arbustives, mosaïques savanes herbeuses / jachères, zones marécageuses, zones artificialisées. Afin d'évaluer l'importance de l'information temporelle, nous avons réalisé différentes combinaisons. Trois classifications de données $\mathrm{S} 2$ ont été réalisées respectivement à partir d'une seule date, de 3 dates puis à partir de 5 dates. De façon analogue, nous avons réalisé deux classifications basées sur les données S1 : à partir d'une

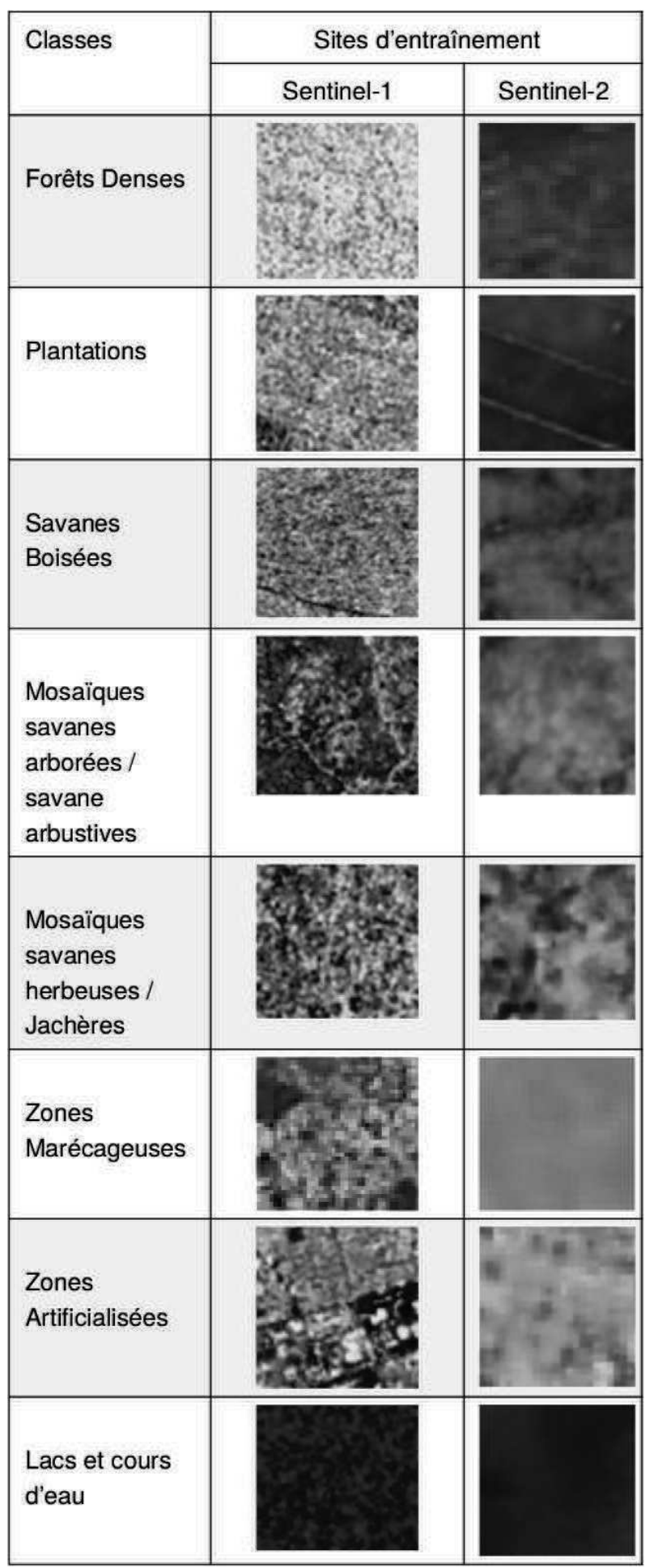

T able 1: Pour chacune des classes, ce tableau illustre la réponse optique ( $R$ : Proche Infra Rouge, $V$ : Moyen Infra Rouge, $B:$ Rouge) et radar ( $R: V V$ en décibels, $V: V H$ en décibels, $B V H$ - VV en décibels).

seule date puis de 33 dates. La grande résolution temporelle des données $\mathrm{S} 1$ nous a permis de produire des profils temporels pour chaque classe d'occupation du sol. La classification a ensuite été pratiquée sur la combinaison des données S1 et S2. 


\subsubsection{Données Sentinel-2}

Les résultats des classifications, détaillés tableau 4.2.1, montrent en moyenne de bons résultats avec une PG de $88,8 \%$ pour l'utilisation d'une scène. Comme attendu, les classes ayant les moins bonnes performances sont les trois classes de savane avec entre $81 \%$ et $88 \%$ de PP. L'ajout de dates supplémentaires montre un apport significatif avec une différence d'environ $3 \%$ à chaque ajout de date. Remarquons que le gain de performance est plus élevé pour les classes de Savanes; probablement car celles ci sont sujettes à de fortes évolutions temporelles.

\subsubsection{Données Sentinel-1}

Les figures 10 (1) et 10 (2) présentent pour chaque classe étudiée l'évolution temporelle des réponses pour les polarisations $\mathrm{VV}$ et $\mathrm{VH}$. Quelle que soit la polarisa-tion considérée, le niveau radiométrique moyen permet de distinguer les classes de formation végétale (forêts et savanes) des classes de formation non végétale (cours d'eau, zones marécageuses, zones artificialisés). De plus, ces profils temporels présentent des variations saison-nières importantes. Les résultats obtenus, détail- lées dans le tableau 2 affichent des résultats satisfaisants avec $85,7 \%$ de $P G$ pour une image $\mathrm{S} 1$. Les classes les moins performantes sont en général les classes ayant les plus grandes densités (à l'exception de la savane boisée). L'utilisation de l'ensemble de la série temporelle (33 dates) permet un gain de performance très important pour arriver à $93,3 \%$ de $P G$. Ce gain s'opère sur l'en-semble de la nomenclature et en particulier pour les mi-lieux qui étaient les moins bien classés avec une seule date

\subsubsection{Combinaison des données Sentinel-2 et Sentinel-1}

Comme le précise le tableau 2, la combinaison des données S2 et S1 apporte un gain significatif de précision globale de $1,9 \%$ puisque l'on passe de $95,2 \%$ et $93,3 \%$, respectivement pour 5 dates $\mathrm{S} 2$ et 33 dates $\mathrm{S} 1$ à $97,6 \%$ en mobilisant les deux capteurs. Quelle que soit la classe considérée (à l'exception de la classe "zones artificialisées") la combinaison S1 S2 fournit des résultats de meilleure qualité. La baisse des performances pour la classe "zones artificialisées" est liée à une forte confusion avec la classe "plantation".

\subsubsection{Conclusion}

L'analyse des résultats obtenus démontre la complémentarité des données $\mathrm{S} 1$ et $\mathrm{S} 2$ dans le contexte de notre étude portant sur la réserve de Togodo au Togo. Ainsi, les images S1 ou S2 exploitées séparément conduisent à des classifications dont les points faibles sont différents, ce qui induit une nette amélioration lors de leur usage conjoint. Ainsi, la combinaison de S1 (33 dates) et S2 (3 dates) permet l'amélioration de l'estimation des surfaces des classes de forêts denses, plantations, savanes boisées, mosaïques savanes arborées- savanes arbustives, mosaïques savanes herbeuses - jachères - cultures qui sont très sensibles aux variations saisonnières. Ces classes sont estimées avec un PP d'environ $98 \%$ contre moins de $90 \%$ pour S1 et environ $90 \%$ pour S2. Cette approche combinant les deux types de capteurs permet de réduire cette contrainte saisonnière par une information plus complète. II est probable que l'écart d'efficacité observé entre capteurs ainsi que leur complémentarité seraient réduits si nous disposions de séries temporelles optiques et radar de grande densité comme cela est discuté dans Inglada et al. (2016). Néanmoins, la disponibilité régulière des données Radar S1 (du fait de leur insensibilité à la couverture nuageuse) permet de les utiliser de façon opérationnelle et récurrente avec l'appui de simplement quelques données optiques. Cette approche est en effet facilement réplicable pour tout type d'écosystème, ce qui constitue ainsi un moyen efficace d'assurer le suivi des différentes classes d'occupation des sols.

\section{Perspectives et conclusion}

Les images gratuites à forte répétitivité aussi bien optiques que radar S1 et S2 élargissent les perspectives d'utilisation de la télédétection pour le suivi de l'occupation du sol. On notera qu'il existe aussi des mosaïques radar en bande L, Palsar et Palsar 2 distribuées gratuitement et particulièrement intéressantes pour leur grande capacité de pénétration mais qui n'ont toutefois pas été évaluées dans le cadre de cette étude.

Les données optiques seules ont montré en moyenne de meilleures performances que les données radar. En revanche les séries temporelles de données radar, de par leur insensibilité à la couverture nuageuse, nécessitent des pré-traitements plus simples à mettre en oeuvre, ce qui permet ainsi d'engager des suivis de changement en quasi temps réel ou des bilans réguliers à l'échelle annuelle. D'une manière générale, la complémentarité des signatures radar et optiques fait que, la combinaison des deux types de données élève leur capacité de discrimination entre les milieux concernés. Cette étude démontre en outre que l'accessibilité des images gratuites Sentinel-1 et Sentinel-2, associée à l'existence d'outils de traitement performants, libres d'accès, autorise leur usage généralisé sur toutes les régions du globe, en particulier tropicales.

\section{Remerciements}

Nous remercions l'ESA pour la fourniture des données radar Sentinel-1 et optique Sentinel-2 gratuites ainsi que le CNES et l'équipe de l'OTB (Orfeo ToolBox) pour le développement de cette boîte à outils de traitement intensivement utilisé pour ce travail. Nous remercions l'ONF pour son appui dans le développement de la méthode de suivi de la déforestation ayant permis le développement du kit d'installation sous Windows et Ubuntu disponible sur demande et via le site www.remotesensing4all.net. Remerciements également au MTES pour l'intérêt qu'il porte au potentiel de l'imagerie radar en contexte tropical. 


\begin{tabular}{|c|c|c|c|c|c|c|c|c|c|c|c|c|}
\hline \multirow[b]{2}{*}{$\begin{array}{c}\text { Classes } \\
\text { thématiques }\end{array}$} & \multicolumn{2}{|c|}{1 date S2 } & \multicolumn{2}{|c|}{3 dates $\mathrm{S} 2$} & \multicolumn{2}{|c|}{5 dates $S 2$} & \multicolumn{2}{|c|}{1 date $\mathrm{S} 1$} & \multicolumn{2}{|c|}{33 date $\mathrm{S} 1$} & \multicolumn{2}{|c|}{$\mathrm{S} 1+\mathrm{S} 2$} \\
\hline & PP & PU & PP & PU & PP & PU & PU & PP & PU & PP & PU & PU \\
\hline Forêts Denses & 89,2 & 91,7 & 90,7 & 94,1 & 96,9 & 97,5 & 72,5 & 81,2 & 94,1 & 97,5 & 99,5 & 100 \\
\hline Plantations & 90,5 & 80,4 & 93,7 & 87,4 & 96,4 & 98,7 & 79,4 & 74,7 & 96,4 & 90,4 & 97,5 & 87,1 \\
\hline Savanes Boisées & 87,5 & 70,7 & 91,9 & 77,3 & 96,8 & 75,4 & 95,8 & 61,2 & 96,8 & 70,7 & 99,5 & 99,4 \\
\hline $\begin{array}{c}\text { Savanes arborées } \\
\text { I savane } \\
\text { arbustives }\end{array}$ & 81,2 & 86,2 & 89 & 90,4 & 81,2 & 95 & 81,2 & 95 & 81,2 & 95 & 99 & 95 \\
\hline $\begin{array}{c}\text { Savanes } \\
\text { herbeuses I } \\
\text { Jachères }\end{array}$ & 81,3 & 97,2 & 84,7 & 97,2 & 90,7 & 99,1 & 88,3 & 99,1 & 88,3 & 99,3 & 96,9 & 99,1 \\
\hline $\begin{array}{c}\text { Zones } \\
\text { Marécageuses }\end{array}$ & 91,3 & 91,6 & 93,9 & 93,3 & 99,9 & 97,9 & 85,7 & 90 & 99,9 & 97,9 & 99,9 & 100 \\
\hline $\begin{array}{c}\text { Zones } \\
\text { Artificialisées }\end{array}$ & 100 & 95,2 & 100 & 98 & 100 & 100 & 91,8 & 93,8 & 100 & 100 & 88,2 & 99,2 \\
\hline $\begin{array}{c}\text { Lacs et cours } \\
\text { d'eau }\end{array}$ & 89,7 & 99,2 & 93,2 & 99,2 & 99,5 & 99,2 & 89,7 & 99,2 & 89,7 & 99,2 & 100 & 100 \\
\hline Précision Globale & \multicolumn{2}{|c|}{88,8} & \multicolumn{2}{|c|}{92,1} & \multicolumn{2}{|c|}{95,2} & \multicolumn{2}{|c|}{85,7} & \multicolumn{2}{|c|}{93,3} & \multicolumn{2}{|c|}{97,6} \\
\hline
\end{tabular}

Table 2 : Statistiques de classification pour différentes combinaison des capteurs S1 et S2 où la combinaison S1+S2 correspond à l'utilisation de toutes les dates S1 et S2

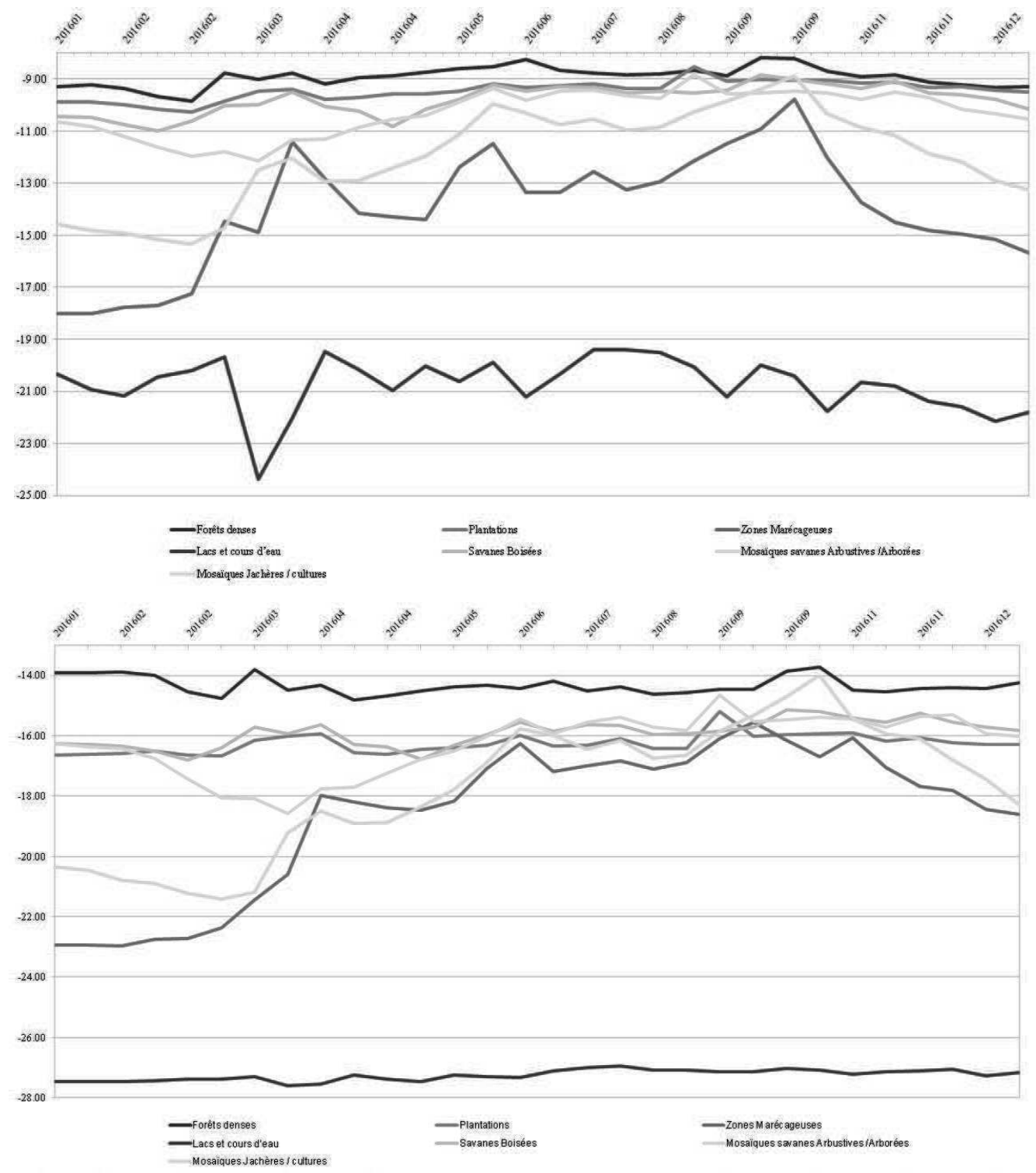

(2)

Figure 10 : Profils temporels pour chacune des classes étudiées en polarisation VV (1) et VH (2). 


\section{Références}

Burges, C. J. C., 1998. A tutorial on support vector machines for pattern recognition. Data Mining and Knowledge Discovery 2, 121-167.

Inglada, J., Vincent, A., Arias, M., Marais-Sicre, C., 2016. Improved early crop type identification by joint use of high temporal resolution sar and optical image time series. $\begin{array}{lllll}\text { Remote Sensing } 8 & \text { (5). URL http:// }\end{array}$ www.mdpi.com/2072-4292/8/5/362

Lardeux, C., Frison, P. L., Tison, C., Souyris, J. C., Stoll, B., Fruneau, B., Rudant, J. P., Dec 2009. Support vector machine for multifrequency sar polarimetric data classification. IEEE Transactions on Geoscience and Remote Sensing 47 (12), 4143-4152.

Maglione, P., Parente, C., Vallario, A., 042016. Pansharpening worldview-2 : Ihs, brovey and zhang methods in comparison Volume 8, 673-679.

Mermoz, S., Le Toan, T., 2016. Forest disturbances and regrowth assessment using alos palsar data from 2007 to 2010 in vietnam, cambodia and lao pdr. Remote Sensing 8 (3).

NIST, 2018. Nist sematech e-handbook of statistical methods ewma control charts.

Olofsson, P., Foody, G. M., Herold, M., Stehman, S. V., Woodcock, C. E., Wulder, M. A., 2014. Good practices for estimating area and assessing accuracy of land change. Remote Sensing of Environment 148, 42 - 57.

Quegan, S., Yu, J. J., Nov 2001. Filtering of multichannel sar images. IEEE Transactions on Geoscience and Remote Sensing 39 (11), 2373-2379. 